\title{
Richtlinien für die Reanimation bei Früh- und Neugeborenen
}

\section{Guidelines for Cardiopulmonary Resuscitation in Preterm and Term Neonates}

Die postnatale Sterblichkeit Früh- und Neugeborener konnte in Deutschland während der vergangenen Dekade deutlich vermindert werden und liegt gemessen im internationalen Vergleich im Spitzenfeld. Wesentlich hierfür sind Fortschritte in der geburtsmedizinischen und neonatologischen Diagnostik und Therapie. Daneben sind strukturelle Maßnahmen, wie z.B. die Zentralisierung von Risikoschwangeren in spezialisierte Einheiten, in diesem Kontext zu erwähnen. Der Qualität der Erstversorgung Früh- und Neugeborener kommt hierbei naturgemäß eine besondere Bedeutung zu, da diese in besonderem Maße die Prognose prägend beeinflusst. Als Beispiele von fast schon historischem Charakter mögen die Vermeidung einer Hypothermie bei Frühgeborenen und Absaugmanöver unter Geburt bei mekoniumhaltigem Fruchtwasser gelten [1].

Zur Umsetzung neuer wissenschaftlicher Erkenntnisse ist das International Liaison Committee on Resuscitation (ILCOR) angetreten, um in einem fünfjährigen Rhythmus entsprechende Richtlinien dem aktuellen Kenntnisstand anzupassen.

Nahezu zeitgleich wurden die Reanimationsrichtlinien der American Association of Pediatrics AAP; [2] publiziert, die nicht in allen Punkten mit den ILCOR-Richtlinien [3] übereinstimmend formuliert sind.

Wesentliche Diskussionspunkte sind unter anderem die Empfehlungen zur Unterlassung des intranatalen Absaugens bei mekoniumhaltigem Fruchtwasser in den ILCOR-Richtlinien, deren Basis im Wesentlichen in einer Studie begründet ist, deren Daten aufgrund relevanter struktureller Unterschiede nicht auf die gegenwärtige Versorgungssituation in der Bundesrepublik Deutschland übertragbar sind [4].

Weitergehende Untersuchungen sind auch hinsichtlich der inspiratorischen Sauerstoffkonzentration im Rahmen einer Primärversorgung nötig, dies gilt besonders für Frühgeborene auch unter dem Aspekt der Entstehung einer Frühgeborenen-Retinopathie [5]. Daneben werden Substanzen, die bei pulmonaler Hypertension sehr früh eingesetzt werden können, wie z. B. Stickstoffmonoxid, nicht in die Überlegungen der Primärversorgung mit einbezogen [6]. Dies sollte bei der Weiterentwicklung entsprechender Richtlinien Berücksichtigung finden.

Weitere Diskussionspunkte mit kritischem Potenzial sind bei der Empfehlung zur initialen Form der maschinellen Beatmung bzw. Masken- beatmung $\mathrm{zu}$ suchen, wo hohe Tidalvolumina, über kurze Zeit tierexperimentell appliziert, ausgeprägte entzündliche Reaktionen nach sich zogen, die für den gesamten Verlauf prägend waren [7].

Somit bleibt festzustellen, dass für Richtlinien in der Primärversorgung dringend weitere Studien benötigt werden, die im Wesentlichen die respiratorische Adaptation sowohl unter der Bedingung der optimalen Sauerstoffversorgung als auch der gewählten Beatmungsdrucke bzw. -volumina bei respiratorischer Insuffizienz zum Gegenstand haben. Auch ist der Einsatz von pulmonal-vasodilatativen Substanzen als weitere Option in diese Primärversorgung oder die danach gelagerte frühe Therapie als Gegenstand weiterer Untersuchungen aktuell.

In diesem Sinne soll der Artikel von Hansmann und Mitarbeitern interpretiert werden, Richtlinien im Rahmen der Primärversorgung nicht als statisches Gebilde, sondern als Herausforderung für neue Studien und als eine kritische Wertung bestehender Daten anzusehen [8]. Ludwig Gortner, Homburg/Saar

\section{Literatur}

1 Keenan WJ: Recommendations for management of the child born through meconium-stained amniotic fluid. Pediatrics 2004; 113: 133-134

2 American Heart Association. 2005 American Heart Association (AHA) guidelines for cardiopulmonary resuscitation (CPR) and emergency cardiovascular care (ECC) of pediatric an neonatal patients: pediatric basic life support. Pediatrics 2006; 117: e989-1004

3 International Liaison Committee on Resuscitation. The International Liaison Committee on Resuscitation (ILCOR) consensus on science with treatment recommendations for pediatric and neonatal patients: pediatric basic and advanced life support. Pediatrics 2006; 117: e955-77

4 Vain NE, Szyld EG, Prudent LM, Wiswell TE, Agular AM, Vivas NI: Oropharyngeal and nasopharyngeal suctioning of meconium-stained neonates before delivery of their shoulders: multicentre, randomised controlled trial. Lancet 2004; 364: 597-602

5 Muller H, Weiss C, Kuntz S, Akkoyun I, Schaible T: Are there predictors for proliferative retinopathy of prematurity and is supplemental oxygen a useful conservative treatment option? Klin. Padiatr 2005; 217: 53-60

6 Hoehn T, Krause MF, Buhrer C: Meta-analysis of inhaled nitric oxide in premature infants: an update. Klin Padiatr 2006; 218: 57-61

7 Kallapur SG, Jobe AH: Contribution of inflammation to lung injury and development. Arch Dis Child Fetal Neonatal Ed 2006; 91: F132-5

8 Hansmann G, Hupl T, Zimmermann A, Bührer C, Wauer $R$, Stannigel $H$, Hoehn $T$ : Neue Reanimationsrichtlinien der ILCOR bei Früh- und Reifgeborenen: Kritische Diskussion und Vorschläge zur praktischen Umsetzung. Klin Padiatr 2007; 219: 51-58 